\title{
Fermi Edge Resonances in Non-equilibrium States of Fermi Gases
}

\author{
E. Bettelheim \\ Racah Institute of Physics, Hebrew University, Jerusalem, Israel \\ Y.Kaplan \\ Racah Institute of Physics, Hebrew University, Jerusalem, Israel \\ P. Wiegmann \\ The James Franck Institute, University of Chicago
}

\begin{abstract}
We formulate the problem of the Fermi Edge Singularity in nonequilibrium states of a Fermi gas as a matrix Riemann-Hilbert problem with an integrable kernel. This formulation is the most suitable for studying the singular behavior at each edge of non-equilibrium Fermi states by means of the method of steepest descent, and also reveals the integrable structure of the problem. We supplement this result by extending the familiar approach to the problem of the Fermi Edge Singularity via the bosonic representation of the electronic operators to nonequilibrium settings. It provides a compact way to extract the leading asymptotes.
\end{abstract}




\section{Introduction}

The FES (Fermi edge singularity) [1, 2, 3, 4] is observed in absorption of X-rays in metals as a power law peak at the Fermi Edge of a degenerate Fermi gas. There a sudden removal of a localized electron from a hard core atomic shell creates a potential which disturbs the electronic gas, thus producing a power low spectrum of electronic soft modes. In recent years the FES has also been demonstrated in tunneling experiments [5, 6, 7, 8]. There a single electron can change the capacity of a contact producing a similar disturbance to the electronic gas as a localized hole. As a result, one observes a power law in tunneling current vs. the bias voltage: $I(V) \sim V^{-2 a+k a^{2}}$ [9], where $\delta=\pi a$ is the scattering phase of the ensuing potential and $k$ is the number of scattering channels. In the case of an attractive potential $(a>0)$ the current peaks at the Fermi edge.

One of the reasons of interest in the FES, and our own motivation in studying it, is that the origin of the FES may be found to be ascribable solely to Fermi statistics. It has been studied over at least five decades, has been well understood, and is considered as one of the fundamental quantum phenomena in electronic physics. Early theoretical papers [1, 2, 3] on the FES and the related phenomenon of Orthogonality Catastrophe [10] were proved to be influential well beyond FES. They are at the foundation of the modern physics of electronic systems in low dimensions.

In a degenerate Fermi gas anything but the leading power asymptotes is rarely of any interest. The reason for that is that everything else, except the leading power, depends on details on band structure, tunneling contacts, etc., and lacks of universal character.

A different situation occurs in a non-equilibrium Fermi gas. There the energy scale of non-equilibrium features can be much smaller than the Fermi scale and can be seen in the spectrum of absorption or tunneling. In this case, additional features become universal.

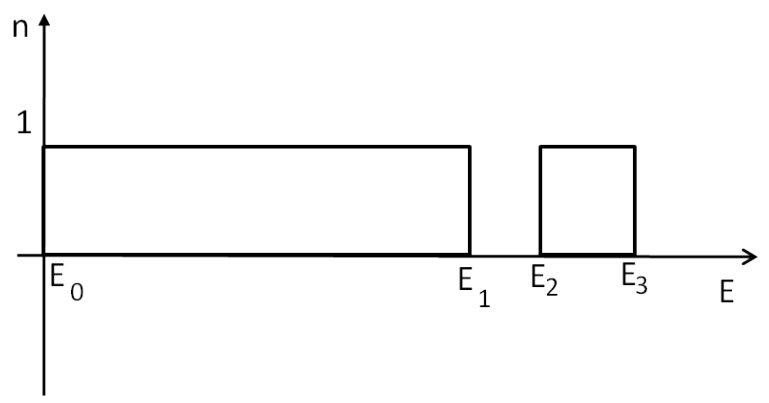

Figure 1. Occupation of electronic states in structured Fermi sea.

As a prototype of a non-equilibrium state we consider a state where the Fermi distribution consists of steps at $E_{1}<E_{2}<\ldots E_{2 n+1}$ such that no states are occupied in the interval between energies $E_{2 i-1}, E_{2 i}$, where $i=1, \ldots, n$. We denote by $E_{0}$ the 
bottom of the filled Fermi sea, which is assumed to be far away Fig. 1. Assuming that the scattering phase does not change within a wide range of the conducting band, say, between $E_{0}=0$ to $E_{2 n}=\Lambda$ the spectrum will be a transcendental universal function of $V / E_{i j}$, where $E_{i j}=E_{i}-E_{j}$.

These structured non-equilibrium states described above inevitably appear in the evolution of an arbitrary semiclassical Fermi state [11. They were also realized in some nanoscale devices (see e.g. [12]).

In a non-equilibrium state the absorption spectrum (or tunneling current) is a transcendental function and elementary methods can determine its asymptotic expansion in various regimes. In this paper we develop a framework aiming to characterize the universal part of the spectrum. We derive a $2 \times 2$ matrix RiemannHilbert problem derived from an inversion problem of an integrable kernel. A similar matrix RH-problems appeared in studies of various fermionic correlation functions and correlation functions of eigenvalues of Random Matrices [13. One of a proven advantage of this formulation is that RH-problem is the most suitable for application of the steepest descent method. The latter prompts the leading asymptotes at Fermi edges. Besides the fact that the RH-problem connects FES to a number of physically unrelated but mathematically equivalent (often well studied) problems, we think that these links and analytical apparatus they bring to physics of the FES are important for a deeper understanding quantum non-equilibrium phenomena. A somewhat alternative, but essentially equivalent approach is to establish a set of non-linear integrable equations [14. We do not discuss this approach here.

As far as the main asymptote is concerned, we will show that in the setting described above the tunneling current at voltage close to a Fermi edge $\varepsilon_{i}\left(e V-E_{i}\right) \ll\left|E_{i, i \pm 1}\right|$ reads:

$$
\begin{aligned}
& I(V) \propto A_{i}\left|e V-E_{i}\right|^{(2 n-1) a^{2}-2 \varepsilon_{i} a} \\
& A_{i}=\mathrm{const} \prod_{l<m}^{2 n} E_{l m}^{2 \varepsilon_{n} \varepsilon_{m} a^{2}} \prod_{0 \leq j \neq i}^{2 n} E_{i j}^{-2 \varepsilon_{j} a}
\end{aligned}
$$
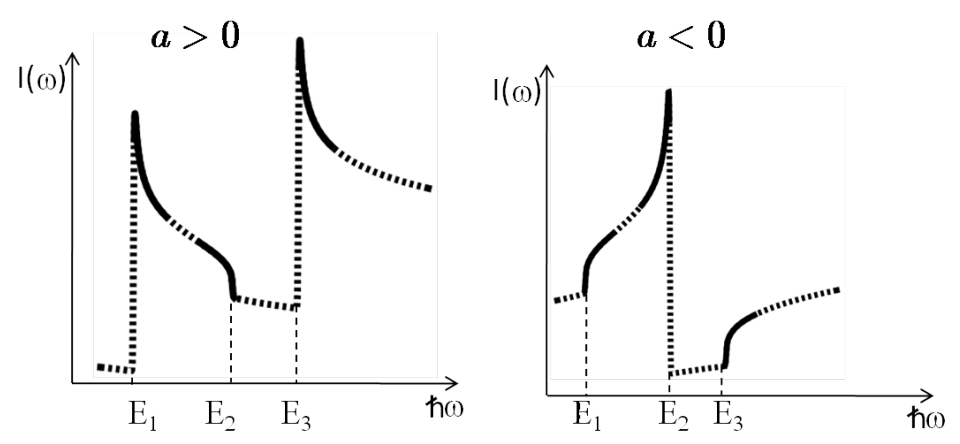

Figure 2. A schematic plot of tunneling current for small $a<0$ (left panel) and $a>0$ (right panel), solid lines show computed power law asymptotes. Dashed lines interpolate between resonances. 
where $\varepsilon_{i}= \pm 1$, if $E_{i}$ is an lower/upper edge of an occupied band, respectively.

Some noticeable features of this result are: (i) the exponent depends on the total number of bands, but stays the same for all upper (lower) edges of occupied intervals $\frac{d I}{I d V}=\left(2 n a^{2}-\left(a-\varepsilon_{i}\right)^{2}\right)\left|V-E_{i} / e\right|^{-1}$; (ii) if the potential is attractive $a>0$ (which is the common case) the current features a peak with a power low decay at upper edges towards increasing energy; and in contrast the current is suppressed at lower edges; if the potential is repulsive, $a<0$, and sufficiently small, a peak appears at the edge $E_{2 k}$ with a power law to the left to the edge Fig.2; (iii) the logarithm of the factor $A_{i}$ can be treated as the electrostatic energy of particles with alternating charges $\pm a$ positioned at the upper/lower edges with an insertion of a unit $\epsilon_{i}$ charge at the edge $E_{i}$.

Fermi Edge singularities with a structured Fermi distribution have been studied before. In 1984 Combescot and Tanguy [15, 16, 17] considered a situation where the interval $\left(E_{1}, E_{2}\right)$ of the band $(0, \Lambda)$ is occupied, while the interval $\left(0, E_{1}\right)$ starting from the bottom of the band is unoccupied. Later 2004 the Abanin and Levitov [18] considered FES with a two steps Fermi distribution. A more general situation has been considered in the recent papers by Gutman et al [19], and by the authors [11], where Eq. (1) have been obtained. The approach employed in these papers (with the exception of Ref.[11]) is based on the expression of the tunneling current as a determinant of a Fredholm operator. A basis of particle-hole excitations of the structured Fermi sea has been used to write the Fredholm operator in early papers [15, 16, 17]. This approach has been developed by Othaka and Tanebe [4] in 1984. Contrary one-particle basis in an empty vacuum has been used in later articles [18, 19]. Naturally the basis of of particle-hole excitations of the Fermi sea, employed in earlier papers [15, 16, 17, 4], which already captures the many-body physics of the Fermi sea is a step forward in obtaining the desired results. We employ this approach here. In addition, for illustrative purposes (Appendix Appendix B), we also present a simple a compact method to capture a leading singularity developed in Ref.[11. That method is based on the bosonic representation of electronic operators in a Fermi state with multiple edges.

\section{Tunneling current.}

The tunneling setting of FES is as follows: a Fermi gas is in contact with a localized resonant level (a quantum dot). It is initially uncharged and provides no scattering to electrons. When an electron tunnels to the dot, it suddenly charges the dot, switchingon a small potential $H \rightarrow H^{\prime}=H+U$ localized at the dot [9]. We assume no further interaction, no dissipation, and we ignore spin and channels.

The tunneling current is given by the golden rule [1, 9]. In units of the tunneling amplitude, $\left.I(\omega)\right|_{\hbar \omega=e V+E_{1}}$ reads

$$
\begin{aligned}
& I(\omega) \propto \operatorname{Re} \int_{0}^{\infty} e^{i \omega\left(t_{1}-t_{2}\right)} G\left(t_{1}, t_{2}\right) d \tau, \\
& G\left(t_{1}, t_{2}\right)=\left\langle\Omega\left|e^{i H t_{2}} c e^{i H^{\prime}\left(t_{1}-t_{2}\right)} c^{\dagger} e^{-i H t_{1}}\right| \Omega\right\rangle
\end{aligned}
$$

Here $c=\sum_{\epsilon} c_{\epsilon}$ is an electronic operator in the position of the dot, assumed to be the 
origin, while $c_{\epsilon}$ is an electronic mode with energy $\epsilon$ and $|\Omega\rangle$ is the structured Fermi state of interest. We also assume that the bias voltage is with respect to $E_{1}$.

Next we assume that the potential is regular within a wide energy range which exceeds the energy range of features of the structured Fermi state and that the tunneling time is sufficiently small. Under this assumption the energy dependence of the scattering phase $\delta$ caused by potential $U$ can be dropped. This amounts to a downwards shift of the energy levels by a constant amount $a$ (in units of level spacing): $\epsilon \rightarrow \epsilon-a$.

In Ref. [3] it has been shown that the vertex operator $e^{a \varphi}$ implements a shift of momenta: the perturbed Hamiltonian and perturbed states are seen as similarity transformation of the unperturbed ones $H^{\prime}=e^{-a \varphi} H e^{a \varphi}$ and $\left|\Omega^{\prime}\right\rangle=e^{a \varphi}|\Omega\rangle$. Here an operator $\varphi$ is a chiral canonical Bose field related to the chiral part of electronic density

$$
\varphi(t)=\hbar \sum_{\epsilon \neq 0} e^{\frac{i}{\hbar} \epsilon t} \rho_{\epsilon} / \epsilon, \quad \rho_{\epsilon}=\sum_{\varepsilon} c_{\varepsilon}^{\dagger} c_{\varepsilon+\epsilon} .
$$

Then Green's function reads

$$
G\left(t_{1}, t_{2}\right)=\left\langle\Omega\left|c\left(t_{2}\right) e^{-a \varphi\left(t_{2}\right)} e^{a \varphi\left(t_{1}\right)} c^{\dagger}\left(t_{1}\right)\right| \Omega\right\rangle,
$$

where $c(t)=\sum_{\epsilon} e^{\frac{i}{\hbar} \epsilon t} c_{\epsilon}$. This formula is standard.

\section{Fredholm Determinants}

Following [1], Green's function can be understood as consisting of three multiplicative factors $G\left(t_{1}, t_{2}\right)=\left|\left\langle\Omega^{\prime} \mid \Omega\right\rangle\right|^{2} e^{C} \cdot L$ - an overall normalization:

$$
\left|\left\langle\Omega^{\prime} \mid \Omega\right\rangle\right|^{2}, \quad \text { where } \quad\left|\Omega^{\prime}\right\rangle=e^{a \varphi(0)}|\Omega\rangle
$$

closed loops

$$
e^{C}=\frac{\left\langle\Omega\left|e^{-a \varphi\left(t_{2}\right)} e^{a \varphi\left(t_{1}\right)}\right| \Omega\right\rangle}{\left|\left\langle\Omega^{\prime} \mid \Omega\right\rangle\right|^{2}},
$$

and open lines

$$
L=\frac{\left\langle\Omega\left|c\left(t_{2}\right) e^{-a \varphi\left(t_{2}\right)} e^{a \varphi\left(t_{1}\right)} c^{\dagger}\left(t_{1}\right)\right| \Omega\right\rangle}{\left\langle\Omega\left|e^{-a \varphi\left(t_{2}\right)} e^{a \varphi\left(t_{1}\right)}\right| \Omega\right\rangle} .
$$

The two latter objects can be cast in the form of Fredholm determinant by means of the Wick theorem. We remind the major formulas.

Consider coherent states of $G l(\infty)$. These states are obtained by transforming the ground state of the Fermi gas $|A\rangle=g(A)|0\rangle$ by an exponent of a bilinear form of Fermi

operators $g(A)=e^{\sum_{\epsilon \eta} A_{\epsilon, \eta} c_{\epsilon}^{\dagger} c_{\eta}}$, where $A_{\epsilon, \eta}$ is an arbitrary $g l(\infty)$ matrix. Our structured Fermi state is a coherent state.

For arbitrary coherent states $A_{1}, A_{2}$ and arbitrary $g l(\infty)$ matrices $B, C$ the following holds

$$
\frac{\left\langle A_{1}|g(B) g(C)| A_{2}\right\rangle}{\left\langle A_{1}|g(B)| A_{2}\right\rangle\left\langle A_{1}|g(C)| A_{2}\right\rangle}=\operatorname{det}(\mathbf{1}+\mathbf{K}),
$$

where

$$
K_{\epsilon_{1}, \epsilon_{2}}=\sum_{\epsilon} M_{\epsilon_{1}, \epsilon}\left(A_{1}, B, A_{3}\right) M_{\epsilon, \epsilon_{2}}^{\dagger}\left(A_{3}, C, A_{2}\right)
$$


and

$$
M_{\epsilon, \eta}\left(A_{1}, B, A_{3}\right)=\left\langle A_{1}\left|g(B) c_{\epsilon}^{\dagger} c_{\eta}\right| A_{3}\right\rangle
$$

are matrix elements of operator $g(B)$ between the state $\langle\Omega(A)|$ and a state $c_{\epsilon}^{\dagger} c_{\eta}\left|A_{3}\right\rangle$, where a particle-hole pair is added to an arbitrary chosen coherent state $\left|A_{3}\right\rangle$. The result does not depend of the choice of $A_{3}$.

In order to obtain this formula one inserts a superposition of an arbitrary number of particle-hole excitations into a chosen coherent state $\left|A_{3}\right\rangle\left\langle A_{3}\right|$, apply the Wick theorem to each term and sum them up.

The variation of $(10) \delta \log \operatorname{det}(\mathbf{1}+\mathbf{K})=\operatorname{tr}\left[(\mathbf{1}+\mathbf{K})^{-1} \delta \mathbf{K}\right]$ gives another known formula

$$
\frac{\left\langle A_{1}\left|c_{\epsilon_{1}}^{\dagger} g(B) g(C) c_{\epsilon_{2}}\right| A_{2}\right\rangle}{\left\langle A|g(B) g(C)| A^{\prime}\right\rangle}=\operatorname{tr}\left[(\mathbf{1}+\mathbf{K})^{-1} \mathbf{P}\right]
$$

where

$$
P_{\epsilon, \eta}\left(\epsilon_{1}, \epsilon_{2}\right)=\tilde{M}_{\epsilon_{1} \eta}\left(A_{1}, B, A_{2}\right) \tilde{M}_{\eta, \epsilon_{2}}^{*}\left(A_{1}, C, A_{2}\right),
$$

and $\tilde{M}_{\epsilon \eta}\left(A_{1}, B, A_{2}\right)=\left\langle A_{1}\left|c_{\epsilon} g(B) c_{\eta}^{\dagger}\right| A_{2}\right\rangle$. Specification of these formulas: $\left\langle A_{1}\right|=\left\langle A_{2}\right|=$ $\langle\Omega|, g(B)=g^{\dagger}(C)=e^{-a \varphi(t)}$ prompts a determinantal representation of the current

$$
e^{C}=\operatorname{det}(\mathbf{1}+\mathbf{K}), L=\operatorname{tr}\left[(\mathbf{1}+\mathbf{K})^{-1} \mathbf{P}\right]
$$

with a kernel

$$
\begin{aligned}
& K\left(\epsilon_{1}, \epsilon_{2}\right)=\sum_{\eta} M_{\epsilon_{1}, \eta}\left(t_{1}\right) M_{\eta, \epsilon_{2}}^{*}\left(t_{2}\right), \\
& P_{\epsilon, \eta}=M_{\epsilon}\left(t_{1}\right) M_{\eta}^{*}\left(t_{2}\right),
\end{aligned}
$$

where

$$
\begin{aligned}
& M_{\epsilon, \eta}(t)=\frac{\left\langle\Omega\left|e^{-a \varphi(t)} c_{\epsilon}^{\dagger} c_{\eta}\right| \Omega\right\rangle}{\left\langle\Omega^{\prime} \mid \Omega\right\rangle}, \\
& M_{\epsilon}(t)=\frac{\left\langle\Omega\left|c(t) e^{-a \varphi(t)} c_{\epsilon}^{\dagger}\right| \Omega\right\rangle}{\left\langle\Omega^{\prime} \mid \Omega\right\rangle}
\end{aligned}
$$

are matrix elements of the vertex operator between states where a particle-hole pair, or just one particle are added to the state $|\Omega\rangle$. These are general formulas valid for any coherent state $|\Omega\rangle$.

The formulas is further specified since the structured Fermi state we are considering is an eigenvalue of the Hamiltonian. In this case

$$
\begin{aligned}
& M_{\epsilon \eta}(t)=e^{\frac{i}{\hbar}(\eta-\epsilon) t} \frac{\left\langle\Omega^{\prime} \mid \Omega ; \epsilon, \eta\right\rangle}{\left\langle\Omega^{\prime} \mid \Omega\right\rangle}, \\
& M_{\epsilon}(t)=e^{-\frac{i}{\hbar} \epsilon t} \frac{\left\langle(\epsilon ; \Omega)^{\prime} \mid \Omega ; \epsilon\right\rangle>}{\left\langle\Omega^{\prime} \mid \Omega\right\rangle} .
\end{aligned}
$$

$M_{\epsilon \eta}$ is an overlap between the state $\left\langle\Omega^{\prime}\right|$ which appears after the shake-up and a particlehole excitation of the state $|\Omega\rangle$ before shake-up with energy $\epsilon \notin \Omega$ and $\eta \in \Omega$, where we $\ddagger$ Formulas equivalent to $\sqrt{16}$ for the ground state can be found in 4 . 
denote $\Omega=\cup_{i=0}^{n-1}\left(E_{2 i}, E_{2 i+1}\right)$ as the set of occupied single particle states in $|\Omega\rangle$, $(\Omega, \epsilon)$ and $(\Omega ; \epsilon, \eta)$ are states where an extra particle or a particle-hole pair is added into $|\Omega\rangle$. Similarly $M_{\epsilon}$ is the overlap of states $|\Omega\rangle$ and the state $\left|\Omega^{\prime}\right\rangle$ with an added particle with energy $\epsilon$.

\section{Matrix elements}

The following formula helps evaluating the matrix elements 20|21): If $\left|\epsilon^{\prime}\right\rangle$ is a single particle eigenstate of the perturbed Hamiltonian and $|\epsilon\rangle$ is a one-particle eigenstate of an unperturbed state then their overlap (in units of level spacing) is $\left\langle\epsilon^{\prime} \mid \epsilon\right\rangle=\frac{\sin (\pi a)}{\pi\left(\epsilon-\epsilon^{\prime}\right)}$. An extension of this formula to many particle states $\langle\underline{\epsilon}|=\left\langle\epsilon_{1}, \ldots, \epsilon_{N}\right|$ and $\left|\underline{\epsilon}^{\prime}\right\rangle=\left|\epsilon_{1}^{\prime}, \ldots, \epsilon_{N}^{\prime}\right\rangle$ gives a Cauchy determinant

$$
\left(\frac{\pi}{\sin (\pi a)}\right)^{N}\left\langle\underline{\epsilon} \mid \underline{\epsilon}^{\prime}\right\rangle=\operatorname{det} \frac{1}{\epsilon_{i}-\epsilon_{j}^{\prime}}=\frac{\prod_{i>j}\left(\epsilon_{i}-\epsilon_{j}\right)\left(\epsilon_{i}^{\prime}-\epsilon_{j}^{\prime}\right)}{\prod_{i, j}\left(\epsilon_{i}^{\prime}-\epsilon_{j}\right)} .
$$

With the help of this formula, the matrix elements in 16 can be computed in a manner similar to Ref. [4]. While computing one must take into account that the set of occupied single particle levels in $\Omega$ and $\Omega^{\prime}$ are shifted by $a$ with respect to each other. Then the problem is reduced to an electrostatic problem of placing a dipole or a charge into a Coulomb plasma confined in the intervals $\cup_{i=1}^{n}\left(E_{2 i}, E_{2 i+1}\right)$. It gives the overlap between states $\langle\Omega|$ and $\left|\Omega^{\prime}\right\rangle$ generalizing Orthogonality Catastrophe formula [10]. Up to an $E_{i}$ -independent constant factor it reads

$$
\left\langle\Omega \mid \Omega^{\prime}\right\rangle \sim \Delta^{(n+1) a^{2}} \prod_{i>j} E_{i j}^{\epsilon_{i} \epsilon_{j} a^{2}} .
$$

The results for matrix elements are

$$
\begin{aligned}
& M_{\epsilon \eta}=e^{\frac{i}{\hbar}(\eta-\epsilon) t} \frac{r(\epsilon) s(\eta)}{\epsilon-\eta}, M_{\epsilon}=e^{\frac{i}{\hbar} \epsilon t} r(\epsilon), \\
& r(\epsilon)=\prod_{i=1}^{2 n}\left(\epsilon-E_{i}\right)^{\varepsilon_{i} a}, \quad \epsilon \notin \Omega ; \\
& s(\eta)=\frac{\sin \pi a}{\pi} \prod_{i=1}^{2 n}\left(E_{i}-\eta\right)^{-\varepsilon_{i} a}, \quad \eta \in \Omega
\end{aligned}
$$

where $\epsilon \notin \Omega, \eta \in \Omega$ are energies of particles and holes. A short sketch of these calculations is found in Appendix Appendix A.

Summing up, the kernel reads

$$
\begin{aligned}
& K\left(\epsilon_{1}, \epsilon_{2}\right)=e^{\frac{i}{\hbar}\left(\epsilon_{2} t_{2}-\epsilon_{1} t_{1}\right)} r\left(\epsilon_{1}\right) r\left(\epsilon_{2}\right) \frac{Q\left(\epsilon_{2}\right)-Q\left(\epsilon_{1}\right)}{\epsilon_{1}-\epsilon_{2}}, \\
& Q(\epsilon, \tau)=\int_{\eta \in \Omega} e^{\frac{i}{\hbar} \eta \tau} \frac{s^{2}(\eta) d \eta}{\epsilon-\eta}, \quad \tau=t_{1}-t_{2} .
\end{aligned}
$$

\section{Integrable kernel}

The next step is to invert the Fredholm kernel $\mathbf{K}$. It can be done in a straightforward manner similar to [4, 15, 16, 17] employing the Wiener-Hopf method at every edge. 
However, calculations become more structured if we use the integrable property of the kernel. Integrability is general property of free fermion correlators (see [20, 21, 22, 13, 14.

A kernel is called integrable if it has the form

$$
K\left(\epsilon_{1}, \epsilon_{2}\right)=\frac{\sum_{\alpha=1}^{l} f_{\alpha}\left(\epsilon_{1}\right) g_{\alpha}\left(\epsilon_{2}\right)}{\epsilon_{1}-\epsilon_{2}}, \quad \sum_{\alpha=1}^{l} f_{\alpha}(\epsilon) g_{\alpha}(\epsilon)=0 .
$$

In the case of a structured Fermi sea $l=2$, and, as follows from (16 24):

$$
\begin{aligned}
& g_{1}(\epsilon)=Q(\epsilon, \tau) g_{2}(\epsilon), \quad f_{2}(\epsilon)=-Q(\epsilon, \tau) f_{1}(\epsilon), \\
& g_{2}(\epsilon)=e^{\frac{i}{\hbar} \epsilon t_{2}} r(\epsilon), \quad f_{1}(\epsilon)=e^{-\frac{i}{\hbar} \epsilon t_{1}} r(\epsilon),
\end{aligned}
$$

Let $\vec{F}=(\mathbf{1}+\mathbf{K})^{-1} \vec{f}$ (we denote $\vec{f}=\left(f_{1}, f_{2}\right)$ ) be a solution of the singular integral equation

$$
\vec{F}\left(\epsilon_{1}\right)+\int_{\epsilon_{2} \notin \Omega} K\left(\epsilon_{1}, \epsilon_{2}\right) \vec{F}\left(\epsilon_{2}\right) d \epsilon_{2}=\vec{f}\left(\epsilon_{1}\right), \quad \epsilon_{1} \notin \Omega .
$$

The time derivative of closed loops contribution and a contribution of open lines (15) are expressed through the solutions $\vec{F}=\left(F_{1}, F_{2}\right)$

$$
\begin{aligned}
& \frac{d C}{d \tau}=\operatorname{tr}\left((\mathbf{1}+\mathbf{K})^{-1} \frac{d \mathbf{K}}{d \tau}\right)=\frac{i}{\hbar} \int_{\epsilon \notin \Omega}\left(g_{1} F_{1}-g_{2} F_{2}\right) d \epsilon \\
& L=\int_{\epsilon \notin \Omega} g_{2} F_{1} d \epsilon,
\end{aligned}
$$

\section{Matrix Riemann-Hilbert problem}

The Fredholm equation (29) is sufficient to obtain the singular behavior at Fermi edges. However, it is instructive to cast the FES problem as a matrix RH problem along the lines described in [13]. In that form, the FES problem falls in the general scheme of integrable problems. In addition, the $\mathrm{RH}$-problem is the most suitable for analysis near edges [20].

The central object of the $\mathrm{RH}$ problem is a matrix-valued functions $m(\epsilon)$ analytic in a complex $\epsilon$-plane cut along the unoccupied intervals $\cup\left(E_{2 i-1}, E_{2 i}\right)$ Fig. 3, defined such that at infinity $m$ approaches the unit matrix, and that its boundary value on the cuts $m_{ \pm}=m(\epsilon \pm i 0)$ connects vector the $\vec{F}$ to the vector $\vec{f}$ as

$$
\vec{F}(\epsilon)=m_{+}(\epsilon) \vec{f}(\epsilon), \quad \epsilon \in \Omega .
$$

In Ref. [13] it has been shown that the matrix is a solution of the RH-problem:

$$
m_{+} v=m_{-}, \quad v_{\alpha \beta}=\delta_{\alpha \beta}-2 \pi i f_{\alpha} g_{\beta} .
$$

In the case of FES

$$
v(\epsilon)=\mathbf{1}+2 \pi i e^{-\frac{i}{\hbar} \epsilon \tau} r^{2}(\epsilon)\left(\begin{array}{cc}
Q & 1 \\
-Q^{2} & -Q
\end{array}\right) .
$$

Eqs (30 34 constitute the matrix RH-problem for FES. As typical for other integrable $\mathrm{RH}$-problems, a similarity transformation can be found to reduce the jump matrix $v(\epsilon)$ 
to a constant matrix, such that analytic behavior in the energy dependence of the kernel will be translated to into the analytic nature of singularities of the solution at infinity. We do not do this here.

Being specified for a one-edge problem $(n=0)$, in units of upper and lower cut-offs, read

$$
\begin{aligned}
& r^{(0)}(\epsilon)=\epsilon^{\varepsilon_{k} a}, \quad \varepsilon_{k} \epsilon>0 \\
& Q^{(0)}(\epsilon, \tau)=\left(\frac{\sin \pi a}{\pi}\right)^{2} \int_{\varepsilon_{k} \eta<0} e^{\frac{i}{\hbar} \eta \tau} \frac{(-\eta)^{-2 \varepsilon_{k} a} d \eta}{\epsilon-\eta},
\end{aligned}
$$

where we count energy from the edge. In this case the RH-problem is solved by elementary means. In fact technically it easier to proceed directly through the integral equation following [4].

\section{Method of steepest descent and the leading singularity}

The asymptotic behavior at the edges can be found by the steepest-descent method described in [20].

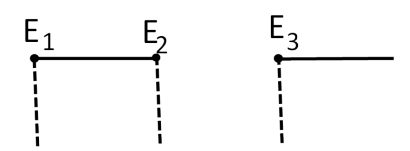

Figure 3. Unoccupied electronic states are between edges $E_{2 k-1}$ and $E_{2 k}, k=1, \ldots n$. The matrix $m$ of the RH-problem (33) jumps on segments of real axis corresponding to unoccupied states (solid line). The steepest descent contour goes down vertically (dashed line) in the lower half plane.

The steepest-descent contour starts from Fermi edges and extends to $i \infty$ in the lower half-plane as is in Fig. 3. Along this contour the rapidly falling exponential factor $e^{-\frac{i}{\hbar} \epsilon \tau}$ in 34 suppresses the jump of the matrix $m$ except at small segments near the Fermi edges, where $r(\epsilon)$ and $Q(\epsilon)$ are singular. At energy close to an edge, say, $E_{i}$ we estimate $r\left(E_{i}+\epsilon\right) \approx r^{(0)}(\epsilon) \prod_{j \neq i}^{2 n} E_{i j}^{\varepsilon_{i} a}$ and $Q\left(E_{i}+\epsilon, \tau\right) \approx e^{\frac{i}{\hbar} E_{i} \tau} \prod_{j \neq i}^{2 n} E_{j i}^{-2 \varepsilon_{i} a} Q^{(0)}(\epsilon)$, and the problem reduces by a similarity transformation to the one-edge problem

$$
\begin{aligned}
& \vec{f}\left(E_{i}+\epsilon\right)=\mathcal{Z}_{i} \vec{f}^{(0)}(\epsilon) ; \quad \vec{g}\left(E_{i}+\epsilon\right)=\mathcal{Z}_{i}^{-1} \vec{g}^{(0)}(\epsilon) ; \\
& m\left(E_{i}+\epsilon\right)=\mathcal{Z}_{i} m^{(0)}(\epsilon) \mathcal{Z}_{i}^{-1} \\
& \mathcal{Z}_{i}=e^{-\frac{i}{2 \hbar} E_{i}\left(\mathbf{1} \cdot t-\sigma_{3} \tau\right)} \prod_{j \neq i}\left|E_{i j}\right|^{\sigma_{3} \varepsilon_{j} a},
\end{aligned}
$$

where script (0) indicates entities of the one-edge problem, $t=t_{1}+t_{2}$, and 1 is the $2 \times 2$ unit matrix.

The reduction of the multi-edge problem to the one edge problem using the $\mathrm{RH}$ steepest descent method is what allows us to solve the problem. Indeed, the contribution of each edge is well known. In units of spacing and up to a constant factor they are

$$
C_{i}^{(0)} \sim \tau^{-a^{2}}, \quad L_{i}^{(0)} \sim \varepsilon_{i} \tau^{2 a-1} .
$$


We note that Ohtaka and Tanebe [4] showed how (38) originally obtained in Refs. [1, 2] by different means) follow from the integral equation (29).

The leading asymptote for the structured Fermi sea can be obtained by a combination of the one edge problem and a similarity transformation. The similarity transformation does not affect the contribution of closed loops. Therefore each edge contributes equally. Summing them up, we obtain $e^{C} \approx e^{\sum_{i} C_{i}^{(0)}}$.

The similarity transformation and (31) give the contribution of open lines as a sum of one edge open lines each weighted by its own amplitude

$$
L=\sum_{i=1}^{2 n+1} e^{-\frac{i}{\hbar} E_{i} \tau} \prod_{j \neq i} E_{i j}^{-2 \varepsilon_{i} a} L_{i}^{(0)}
$$

Combining the normalization the closed loop and the open line we obtain:

$$
G(\tau) \sim \tau^{-(2 n+1) a^{2}}\left(\prod_{i<j} E_{i, j}^{2 \varepsilon_{i} \varepsilon_{j} a^{2}}\right) e \sum_{m} \varepsilon_{m} \tau^{2 a-1} e^{-\frac{i}{\hbar} E_{m} \tau} \prod_{n \neq m} E_{m, n}^{-2 \varepsilon_{m} a},
$$

where a multiplicative constant depending only on cutoffs has been omitted. Fourier transforming, this formula prompts the main result (1).

\section{Acknowledgment}

The authors acknowledge discussions with A. Mirlin, D. Gutman, S.-Y. Lee and especially A. Abanov. P. W. was supported by NSF DMR-0906427, MRSEC under DMR-0820054. E. B. was supported by grant 206/07 from the ISF.

\section{Appendix A. Compnutation of 23,24}

We sketch the computations of matrix element (23). They are not much different from similar calculations for a single edge problem [4]. We start from a general formula (22) describing an overlap of arbitrary electronic states consisting of a finite number $N$ of occupied levels. It can be seen as the exponent of the electrostatic interaction energy of log-interacting particles with charges of size $+a$ at $\epsilon_{i} \in \Omega$ 's and $-a$ at $\epsilon_{i}^{\prime} \in \Omega-a$. Thus

we have a regular alternating pattern of charges, the negative charges at an offset of $-a$ with respect to the positive ones, the patten starts at $E_{0}$ and persists up to $E_{2 n+1}$, with gaps at $\left[E_{2 i-1}, E_{2 i}\right], i=1, \ldots, n$. The electrostatic energy is:

$$
\sum_{i \neq j, \epsilon_{i}, \epsilon_{j} \in \Omega} \log \left(\frac{\epsilon_{i}-\epsilon_{j}}{\epsilon_{i}-\epsilon_{j}+a}\right)-N \log a .
$$

This sum can be evaluated in terms of Barnes functions, but we are interested only in the limit of large number of levels. There we may think in terms of a density of dipoles with a polarization vector $a$. The energy of this system is equivalent to energy of charges $\varepsilon_{i} a$ placed at edge $E_{i}$. The latter (up to $a$-dependent constant) is $\sum_{i<j} \varepsilon_{i} \varepsilon_{j} a^{2} \log \left|E_{i}-E_{j}\right|$. The exponent of the energy yields 23 .

The Configuration of charges corresponding to $M_{\epsilon, \eta}$ is a set of dipoles discussed above minus a charge +1 at $\epsilon \in \Omega$ plus a charge -1 at $\eta \notin \Omega$. The energy acquires the 
interaction energy between charges at $\eta$ and $\epsilon$ and charges $\pm a$ sitting at edges $E_{i}$. This addition is $\sum_{i} \varepsilon_{i} a\left(\log \left|\epsilon-E_{i}\right|-\log \left|\eta-E_{i}\right|\right)$ plus an $a$ dependent constant. The origin of the constant is the energy of dipoles in the vicinity of a hole at $\epsilon$. It is $\sum_{\epsilon_{i} \in \Omega} \log \frac{\epsilon_{i}-\eta-a}{\epsilon_{i}-\eta}$. Assuming that $\eta$ is far from edges the contribution goes from levels close to $\epsilon$. It gives $\log \left(\frac{\sin (\pi a)}{\pi}\right)$. All together it yields 24 . Computation of $M_{\epsilon}$ is similar. In that case only interactions between the extra charge at $\epsilon \notin \Omega$ and residual charges at edges contribute. It does not incur a constant factor.

\section{Appendix B. Computation through bosonic representation}

The leading singularity can be understood using a bosonic formalism. First we separate fast oscillatory modes at each edge

$$
c\left(t, x_{0}\right)=\sum_{i} e^{\frac{i}{\hbar} E_{i} t} \psi_{i}(t) .
$$

Then we represent slow modes through components of the Bose field $\partial_{x} \varphi_{i}=i \psi_{i}^{\dagger}(t) \psi_{i}(t)$ as

$$
\psi_{i} \propto\left(\varepsilon_{i} \prod_{j \neq i} E_{i j}^{\varepsilon_{j}}\right)^{1 / 2} e^{-\varepsilon_{i} \varphi_{i}} .
$$

The Bose field (5) is a sum of its components $\varphi=\sum_{k} \varphi_{i}$. Components of the Bose field represent particle-holes excitations close at each edge. At $\hbar / \tau \gg E_{i j}$ they can be treated as independent canonical Bose fields. Their variances $D_{i}\left(t_{1}, t_{2}\right)=$

$-\frac{1}{2}\left\langle\Omega\left|\left(\varphi_{i}\left(t_{2}\right)-\varphi_{i}\left(t_{1}\right)\right)^{2}\right| \Omega\right\rangle$ are not difficult to compute. As follows form (5), $D_{2 k-1}$ are sums of $(\cos \epsilon \tau-1) / \epsilon$ over all possible energy of a particle-hole excitations provided that a particle is placed in the "gap" $\left(E_{2 k-1}, E_{2 k}\right)$. Similarly $D_{2 k}$ is the sum over energies of a hole-particle excitations provided that a hole is placed to the band $\left(E_{2 k}, E_{2 k+1}\right)$. Computing these integrals at $\tau \gg \hbar /\left|E_{i j}\right|$ one obtains

$$
D_{i}(\tau)=-\log \tau+\varepsilon_{i} \sum_{j \neq i} \varepsilon_{j} \log \left|E_{i j}\right|
$$

The time independent term in (B.2) explains the prefactor in B.1): the correlator $\left\langle\psi_{i}^{\dagger}\left(t_{1}\right) \psi_{i}\left(t_{2}\right)\right\rangle \propto \frac{\varepsilon_{i}}{\tau}$ then also has to be obtained for the Bose field. This yields $\left(\prod_{j \neq i} E_{i j}^{-\varepsilon_{i} \varepsilon_{j}}\right) e^{D_{i}}$.

In the Bose representation, Green's function (6) is a sum of edge components

$$
\begin{aligned}
& G\left(t_{1}, t_{2}\right)=\sum_{i}\left(\varepsilon_{i} \prod_{j \neq i} E_{i j}^{-\varepsilon_{i} \varepsilon_{j}}\right) e^{\frac{i}{\hbar} E_{i} \tau} G_{i}\left(t_{1}, t_{2}\right), \\
& G_{i}=\left\langle e^{\left(\varepsilon_{i}-a\right)\left(\varphi_{i}\left(t_{2}\right)-\varphi_{i}\left(t_{1}\right)\right)}\right\rangle \prod_{j \neq i}\left\langle e^{-a\left(\varphi_{j}\left(t_{2}\right)-\varphi_{j}\left(t_{1}\right)\right)}\right\rangle
\end{aligned}
$$

Computing this, we obtain Green's function 400.

\section{References}

[1] P. Nozières and C. T. de Dominicis. Singularities in the X-Ray Absorption and Emission of Metals. III. One-Body Theory Exact Solution. Physical Review, 178:1097-1107, 1969. 
[2] G. D. Mahan. Excitons in Metals: Infinite Hole Mass. Physical Review, 163:612-617, 1967.

[3] K. D. Schotte and U. Schotte. Tomonaga's Model and the Threshold Singularity of X-Ray Spectra of Metals. Physical Review, 182:479-482, 1969.

[4] K. Ohtaka and Y. Tanabe. Theory of the soft-x-ray edge problem in simple metals: historical survey and recent developments. Reviews of Modern Physics, 62:929-992, October 1990.

[5] A. K. Geim, P. C. Main, N. La Scala, Jr., L. Eaves, T. J. Foster, P. H. Beton, J. W. Sakai, F. W. Sheard, M. Henini, G. Hill, and M. A. Pate. Fermi-edge singularity in resonant tunneling. Physical Review Letters, 72:2061-2064, March 1994.

[6] D. H. Cobden and B. A. Muzykantskii. Finite-Temperature Fermi-Edge Singularity in Tunneling Studied Using Random Telegraph Signals. Physical Review Letters, 75:4274-4277, December 1995.

[7] I. Hapke-Wurst, U. Zeitler, H. Frahm, A. G. M. Jansen, R. J. Haug, and K. Pierz. Magnetic-fieldinduced singularities in spin-dependent tunneling through InAs quantum dots. Phys. Rev. B, 62:12621-12624, November 2000.

[8] Y. N. Khanin, E. E. Vdovin, L. Eaves, I. A. Larkin, A. Patane, O. N. Makarovskii, and M. Henini. Magnetic-field-induced Fermi-edge singularity in the tunneling current through an InAs selfassembled quantum dot. Soviet Journal of Experimental and Theoretical Physics, 105:152-154, July 2007.

[9] K. A. Matveev and A. I. Larkin. Interaction-induced threshold singularities in tunneling via localized levels. Phys. Rev. B, 46, 1992.

[10] P. W. Anderson. Infrared Catastrophe in Fermi Gases with Local Scattering Potentials. Physical Review Letters, 18:1049-1051, June 1967.

[11] E. Bettelheim, Y. Kaplan, and P. B. Wiegmann. Gradient Catastrophe and Fermi Edge Resonances in Fermi Gas. ArXiv e-prints/1011.1993, November 2010.

[12] S. de Franceschi, R. Hanson, W. G. van der Wiel, J. M. Elzerman, J. J. Wijpkema, T. Fujisawa, S. Tarucha, and L. P. Kouwenhoven. Out-of-Equilibrium Kondo Effect in a Mesoscopic Device. Physical Review Letters, 89(15):156801-+, September 2002.

[13] P. Deift, A. Its, and X. Zhou. A Riemann-Hilbert approach to asymptotic problems arising in the theory of random matrix models, and also in the theory of integrable statistical mechanics. Ann. of Math., 146:149-235, 1997.

[14] E. Bettelheim, A. G. Abanov, and P. B. Wiegmann. FAST TRACK COMMUNICATION: Quantum hydrodynamics and nonlinear differential equations for degenerate Fermi gas. Journal of Physics A Mathematical General, 41:2003-+, October 2008.

[15] M. Combescot and C. Tanguy. Absorption-edge singularities for a nonequilibrium Fermi sea. I. Second-order perturbation theory. Phys. Rev. B, 50:11484-11498, October 1994.

[16] C. Tanguy and M. Combescot. Absorption-edge singularities for a nonequilibrium Fermi sea. II. Second-order diagrammatic expansion. Phys. Rev. B, 50:11499-11507, October 1994.

[17] C. Tanguy and M. Combescot. Absorption-edge singularities for a nonequilibrium Fermi sea. III. Determinantal nonperturbative theory. Phys. Rev. B, 52:11698-11710, October 1995.

[18] D. A. Abanin and L. S. Levitov. Tunable Fermi-Edge Resonance in an Open Quantum Dot. Physical Review Letters, 93(12):126802-+, September 2004.

[19] D. B. Gutman, Y. Gefen, and A. D. Mirlin. Non-equilibrium 1D many-body problems and asymptotic properties of Toeplitz determinants. ArXiv e-prints, October 2010.

[20] P. Deift and X. Zhou. A steepest descent method for oscillatory Riemann-Hilbert problems. ArXiv Mathematics e-prints, December 1992.

[21] M. Jimbo, T. Miwa, Y. Môri, and M. Sato. Density matrix of an impenetrable Bose gas and the fifth Painlevé transcendent. Physica D Nonlinear Phenomena, 1:80-158, April 1980.

[22] A. R. Its, A. G. Izergin, V. E. Korepin, and N. A. Slavnov. Differential Equations for Quantum Correlation Functions. International Journal of Modern Physics B, 4:1003-1037, 1990. 